\title{
Role of Magnetic Resonace Imaging in Patients with Chronic Wrist Pain
}

\author{
Authors \\ Dr Ekta Vinubhai Vadher ${ }^{1}$, Dr Tosha Jatin Desai ${ }^{2}$, Dr Nandini Umesh Bahri ${ }^{3}$ \\ Corresponding Author \\ Dr Ekta Vinubhai Vadher \\ Pratik', Silvershine Society, Nana-Mava Road, Rajkot, Gujarat, India
}

\begin{abstract}
Background: The wrist is a complex joint that bridges the hand to the forearm. Chronic wrist pain causes disabilities in day to day activities. Clinical examination helps in assessment, but for further evaluation, imaging provides detailed information and helps in patient's management.

Aim of the study is to evaluate post-traumatic lesions, avascular necrosis, chondral injuries and arthritis, tendon pathologies and internal derangements of ligaments and the triangular fibrocartilage complex (TFCC) by using magnetic resonance imaging technique.

Material and Methods: A prospective study, was carried out in our institute from August 2014 to October, 2016, with written approval of ethical committee, under which 50 patients were selected who were referred from orthopaedic department with complaint of chronic (>6 months) wrist pain and positive tests in clinical examination, assessed by using SIEMENS MAGNETOM ESSENZA 1.5T MRI machine for characterization.

Results: In our study, most common pathology causing chronic wrist pain was ganglionic cyst (56\%) followed by tendon pathology (36\%), infective/inflammatory conditions (22\%), ligamentous degenerative tear (18\%), and osseous tumour (4\%). Most common ligamentous injury is triangular fibro-cartilage complex (TFCC).

Conclusion: Magnetic resonance imaging provides a relevant way to diagnose the wrist pathologies with high-resolution, multi-planar imaging without employing ionizing radiation. It influences clinical and surgical diagnosis and management of wrist pathologies.

Keywords: Chronic wrist pain, Magnetic resonance imaging, Triangular fibro-cartilage complex (TFCC).
\end{abstract}

\section{Introduction}

The wrist is a complex joint that bridges the hand to the forearm. It is actually a collection of multiple bones and joints. The bones comprising the wrist include the distal ends of the radius and ulna, 8 carpal bones, and the proximal portions of the 5 metacarpal bones. They support a tube that runs through your wrist. That tube, called the carpal tunnel, has tendons and a nerve inside. It is covered by a ligament, which holds it in place. ${ }^{1,2,3,4,5}$
Pain is common symptom for wrist joint. Repetitive motion can damage your wrist. Everyday activities like typing, racquet sports or sewing can cause pain, or even carpal tunnel syndrome.

Clinical examination is done to localize and identify the painful structures through systematic palpation of the wrist's bony and soft-tissue anatomy and to perform provocative tests to reproduce the patient's symptoms and identify instability. ${ }^{6,7}$ It includes inspection for any redness, hypertrophy or atrophy of hypothenar or thenar group of muscles, range of 
motion and various tests like finkelstein's test (for DeQuervain's tenosynovitis), watson test (for scapholunate instability), lunotriquestralballotment (Reagan test) (for lunotriquestral instability), kleinman shear test (for LT instabiliy), lischtan test (for mid carpal instability), TFCC grind test (for TFCC pathology), ECU snap test (for ECU instability), Piano key test (for DRUJ instability), Fovea sign (for lunotriquetral ligament split tear). Furthermore, tinel, phalen tests are used for the carpal tunnel syndrome. ${ }^{8}$

Various imaging modalities are used to diagnose cause of pain. Plain radiograph is primary modality of imaging. It helps for evaluation of bony abnormalities. However, many of times they are unremarkable, either due to ligamentous or tendon involvement or due to early course of disease. Patients with non-resolving chronic wrist pain even with medications and positive clinical findings are the candidates who require further advanced cross sectioned imaging.

Magnetic resonance imaging being high-resolution, multiplanar imaging technique without employing ionizing radiation, is helpful for evaluation of various ligaments, tendons, carpal tunnel, articular cartilage and bone marrow. ${ }^{9}$

\section{Objective}

1. To evaluate various causes of chronic wrist pain in patients with positive clinical examination through magnetic resonance imaging technique.

2. To know most common causes of chronic wrist pain.

\section{Material and Methods}

This is a prospective observation study.

Study is carried out in our institute with 1.5 tesla, Siemens MEGNATONESSENZA machine.

\section{Study Settings}

The study was conducted our institute during a period from August, 2014 to October, 2016 with written approval of ethical committee and standard procedures.

\section{Inclusion Criteria}

- Age : various age group

- Sex : male/ female

- Patient presenting to the orthopaedic OPD with complaint of chronic (6 months) wrist pain with positive clinical examination who may or may not have other complains like swelling and limitation of movements.

\section{Exclusion Criteria}

- Traumatic injuries.

- Wrist pain for $<6$ months.

- Negative clinical examination.

- Patients with other contraindications for MRI like, Cardiac Pacemaker, Metallic implants, Claustrophobia, Non co-operative patient, hemodynamically unstable patient.

\section{Sample Size}

A total of 50 patients having chronic wrist pain were included.

The Routine MRI Sequences Applied were T1WI, T2WI, PDFS, 3D SPGR and STIR sequences were obtained in the axial, coronal, and sagittal planes. Coronal images are acquired with 2- to $2.5-\mathrm{mm}$ sections, using a $6-\mathrm{cm}$ FOV and a $512 \times 256$ or 256 $\times 256$ matrix.

\section{Assessment of MRI Wrist}

- Pathology of the TFC and intrinsic ligaments is displayed on FS PD FSE coronal images, which create an arthrography-like effect by displaying the hyperintensity of fluid in contrast to the lower signal intensity of ligaments and fibrocartilage. (The sequence also used in the axial and sagittal planes.)

- T2\&ast;-weighted coronal images produces excellent contrast between ligaments (the intercarpal ligaments and the TFC complex) and fluid. Intrasubstance TFC degeneration is best demonstrated in this sequence.

- 3D SPGR techniques are used to display detailed anatomy of the TFC complex and intrinsic ligaments. Using these sequences, it is possible to achieve higher-resolution MR images. FS is recommended.

- An axial STIR or FS PD FSE sequence demonstrates tenosynovitis, ganglions, 
carpal tunnel syndrome and related changes in the median nerve, and neoplasms. The distinct dorsal, membranous, and volar components of the scapholunate ligament are separated on axial images through the proximal carpal row. The flexor digitorumsuperficialis and profundus tendons can be differentiated on gradient echo axial images through the phalanges. 10

\section{Observations}

Following observations were made on the basis of MRI wrist-

Table 1. Age distribution

Most of the wrist pathologies were reported in individuals between 20-29 years of age $(n=18,36 \%$ of study population) followed by $30-39$ years $(n=$ $26 \%$ ).

\begin{tabular}{|l|c|c|}
\hline $\begin{array}{l}\text { Age group (in } \\
\text { years) }\end{array}$ & $\begin{array}{c}\text { No. of Patients } \\
(\mathrm{n}=50)\end{array}$ & $\begin{array}{c}\text { Percentage of } \\
\text { Patients (\%) }\end{array}$ \\
\hline $0-9$ & 1 & 2 \\
\hline $10-19$ & 3 & 6 \\
\hline $20-29$ & 18 & 36 \\
\hline $30-39$ & 13 & 26 \\
\hline $40-49$ & 7 & 14 \\
\hline $50-59$ & 4 & 8 \\
\hline $60-69$ & 4 & 8 \\
\hline
\end{tabular}

Table 2. Frequency of wrist pathologies on MRI

\begin{tabular}{|l|c|c|}
\hline & $\begin{array}{c}\text { No. of Patients } \\
(\mathrm{n}=50)\end{array}$ & $\begin{array}{c}\text { Percentage of } \\
\text { Patients (\%) }\end{array}$ \\
\hline Ligaments injury & 9 & 18 \\
\hline osteomyelitis & 6 & 12 \\
\hline Osseous tumours & 2 & 4 \\
\hline Pathologies of tendons & 18 & 36 \\
\hline Ganglionic cyst & 28 & 56 \\
\hline Arthritis & 5 & 10 \\
\hline
\end{tabular}

Most common pathology affecting wrist was ganglionic cyst. Here note that these groups might be seen in same patients, like ligamentous degenerative tear might be seen associated with arthritis.

Table 3 Ligamentous injuries

\begin{tabular}{|l|c|c|}
\hline Ligamental Injuries & $\begin{array}{c}\text { No. of Patients } \\
(\mathrm{n}=9)\end{array}$ & $\begin{array}{c}\text { Percentage of } \\
\text { Patients (\%) }\end{array}$ \\
\hline $\begin{array}{l}\text { Triangular fibro-cartilage } \\
\text { complex }\end{array}$ & 8 & 88.89 \\
\hline Scapholunate ligament & 2 & 22.22 \\
\hline Lunotriquetral ligament & 1 & 11.11 \\
\hline
\end{tabular}

Out of 50, 9 patients were having Ligamentous abnormality. Among ligament injuries, most common ligament group involved was triangular fibro-cartilage complex, followed by lunotriquetral ligament and scapholunate ligament.

Figure 1.Tendon pathology according to MRI

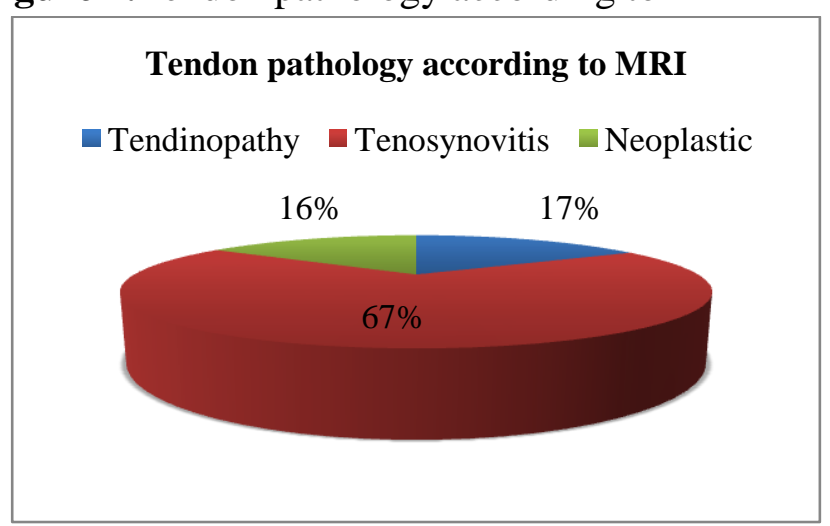

Most common tendon pathology was tenosynovitis.

Figure 2: Sex distribution of wrist pathology

In present study, we have concluded that $40 \%$ of wrist pathologies occur in males while $60 \%$ affect females. Hence, a slight preponderance for female gender as compared to male gender in wrist pathologies is noted. (Female: Male ratio $=3: 2$ ).

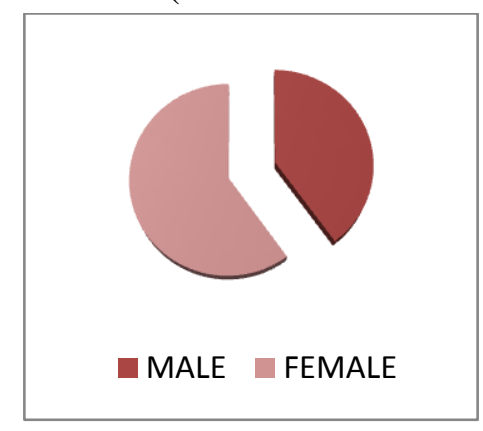

Figure 3.Dorsal Ganglionic Cyst

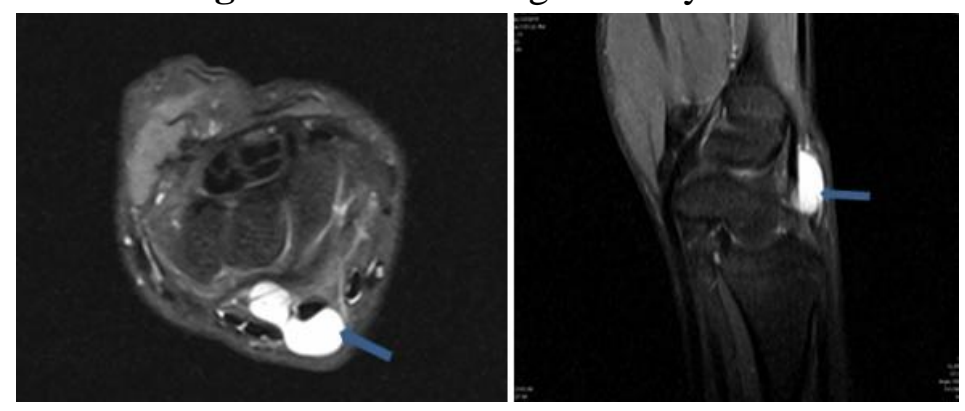

A well-defined, lobulated cystic lesion at the scapholunate region on dorsal aspect of wrist. It traversing through the extensor digitorumlongus tendons. 
Figure 4. Dequervain's Tenosynovitis
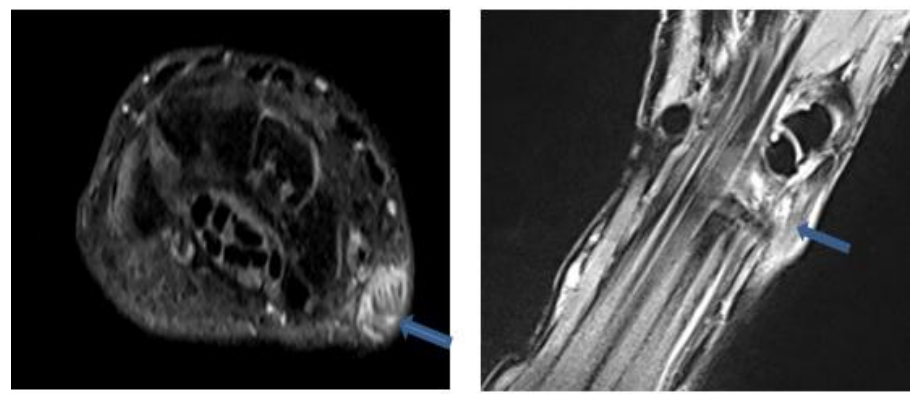

First extensor compartmental tendons (abductor pollicuslongus and extensor pollicusbrevis) appear bulky and show increased signal intensity.

Figure 5.Tenosynovial Chondromatosis

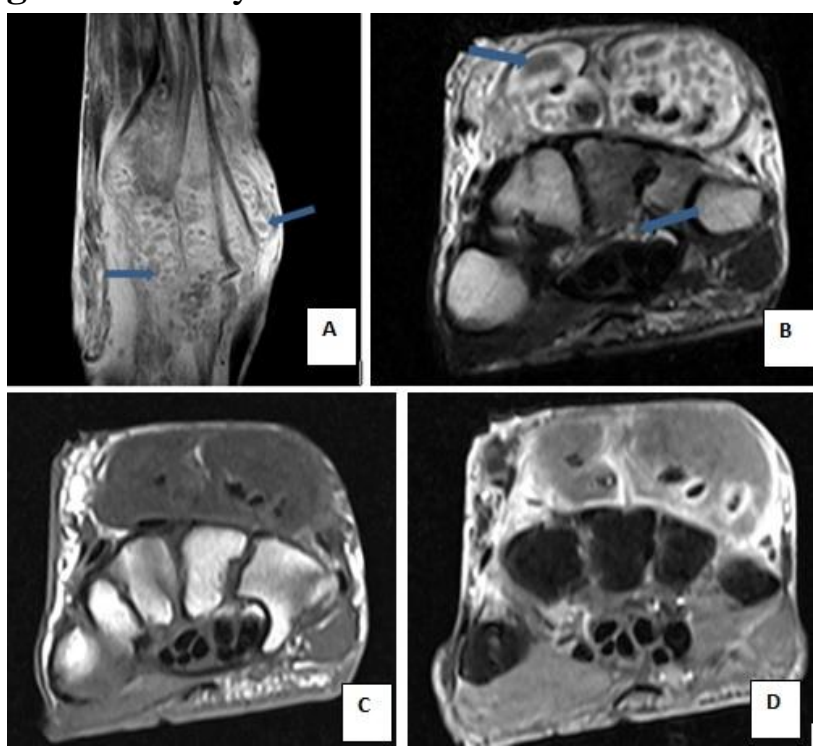

Image $\mathrm{A}$ and $\mathrm{B}$ show rice bodies in flexor and extensor tendon sheaths; in flexor part it lies deep to carpal tunnel tendons in palm. Images $\mathrm{C}$ and $\mathrm{D}$ are pre- and post- contrast T1FS images which show post contrast synovial enhancement.

Figure 6.Giant Cell Tumour Of Ulna
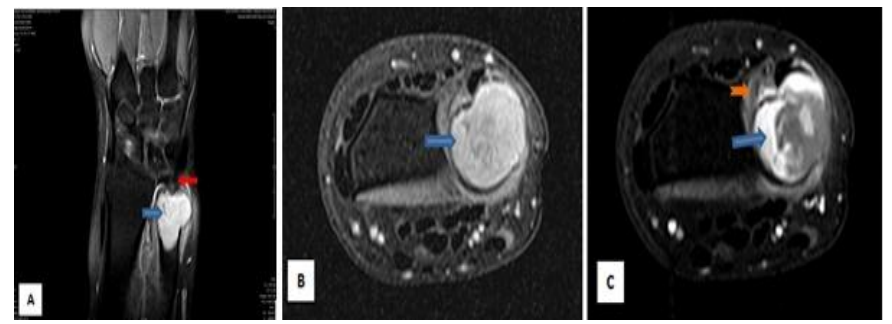

MRI- IMAGE A (PDFS), B (T1FS) and C (T1FSPOSTCONT) - altered signal intensity lesion (arrow) with surrounding enhancing soft tissue (notched arrow) and resultant increased signal intensity involving TFCC (red arrow)

Figure 7.Chondroblastoma

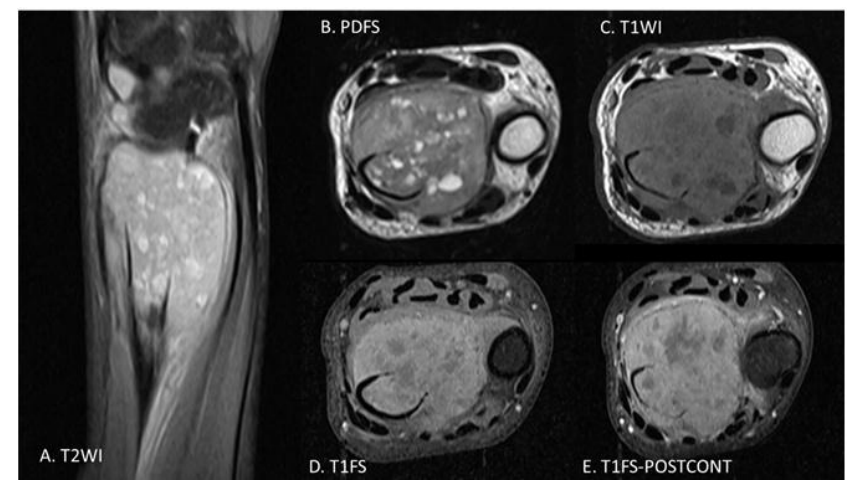

Contrast MRI- a lytic, expansile, heterogeneously enhancing soft tissue lesion involving radio-ulnar syndesmosis. However, no evidence of invasion of surrounding tendons or vessels. Bone marrow appears unremarkable.

Figure 8.Kien Bock Disease

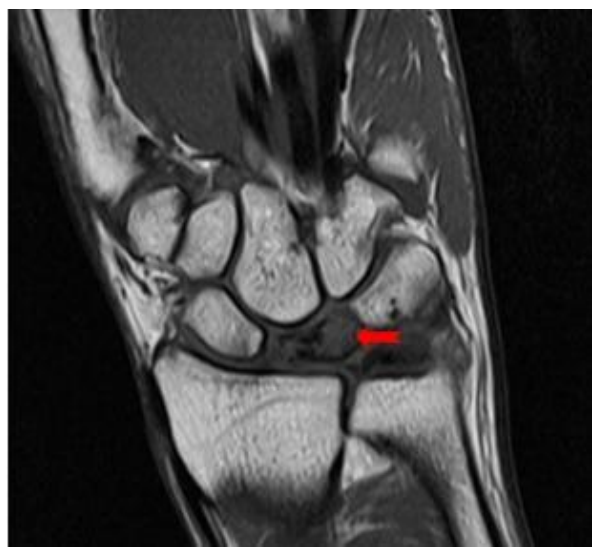

Grade III osteonecrosis of lunate with resultant partial collapse, fragmentation and variegated contrast enhancement pattern (low viability of bone) with negative ulnar variance.

\section{Discussion}

Chronic wrist pain is clinically difficult to evaluate and manage. Patients have often suffered long-term pain, sought advice from many medical sources with no effect. Often the specific diagnosis is not known, in spite of positive clinical examination. Though a large number of aetiologies are associated with the chronic painful wrist, very little information is available regarding causation and diagnosis with the literature dominated by the 
diagnosis of pannus and synovitis associated with rheumatoid arthritis. Some aetiologies such as the dorsal occult ganglion have been assessed with MRI 11,12 . The natural history of undiagnosed wrist pain, particularly in the young, may be disabling, with long term follow up. A subset of ganglia are thought to cause chronic pain secondary to or related to internal derangements of the wrist, ligament tears, tenosynovitis, tendinopathies, avascular necrosis and arthritis. ${ }^{13,14}$ The significance of being able to distinguish between the these entities is important, and preoperative MRI may allow a specific diagnosis. ${ }^{15}$

In our study, 50 patients with chronic wrist pain were evaluated. Maximum numbers (18\%) of patients were between 20-29 years. This shows that it is quite common in younger age group. Females were more commonly affected than males.

Most common cause for chronic wrist pain was ganglionic cyst. Triangular fibro-cartilage complex was most commonly involved ligament for degenerative tear. Most commonly involved tendons where extensors and most common tendon pathology was tenosynovitis.

Other causes of wrist pain were arthritis, osteomyelitis, avascular necrosis and osseous tumours, which were in lesser frequency.

\section{Conclusion}

For patients with chronic wrist pain causing disability with positive clinical examination, MRI helps in diagnosing pathology more accurately. MRI is especially well suited for evaluation of the small soft tissue structures of the wrist, including the tendons, the intrinsic ligaments and the neurovascular structures. It is also accurately depicts abnormalities of the osseous structures including osteomyelitis, avascular necrosis and bony neoplasms.

\section{Acknowledgment}

We are thankful to Dr.H.P.Parekh (M.D, Professor), Dr.S.L.Chudasama (M.D, Associate Professor) for their inputs on the subject and Mr.Sanghani, our skilled technician staff.

\section{References}

1. Al Saffar RA. Quantification of the variable radiocarpal ligaments pattern (Doctoral dissertation, University of Glasgow).

2. Nichols JA, Bednar MS, Murray WM. Orientations of wrist axes of rotation influence torque required to hold the hand against gravity: A simulation study of the nonimpaired and surgically salvaged wrist. Journal of biomechanics. 2013 Jan 4;46(1):192-6.

3. Iida A, Omokawa S, Moritomo H, Aoki M, Wada T, Kataoka T, Tanaka Y. Biomechanical study of the extensor carpi ulnaris as a dynamic wrist stabilizer. The Journal of hand surgery. 2012 Dec 31;37(12):2456-61.

4. Tang JB, Chen YR. In vivo changes in contact regions of the radiocarpal joint during wrist hyperextension. The Journal of hand surgery. 2012 Nov 30;37(11):2257-62.

5. Halaki M, O'Dwyer N, Cathers I, Heritier S. Systematic nonlinear relations between joint mechanics and the neural reflex response with changes in stretch amplitude at the wrist. Journal of biomechanics. 2012 Nov 15;45(16):2755-62.

6. Abdelmegeed MA. Physical Therapy after Triangular Fibrocartilage Injuries and Ulnar Wrist Pain.

7. Magee DJ. Orthopedic physical assessment. Elsevier Health Sciences; 2014 Mar 25.

8. Cevik AA, Gunal I, Manisali M, Yanturali S, Atilla R, Pekdemir M, Gunerli A, Holliman CJ. Evaluation of physical findings in acute wrist trauma in the emergency department. Ulusaltravmaveacilcerrahidergisi $=$ Turkish journal of trauma \& emergency surgery: TJTES. 2003 Oct;9(4):257-61.

9. Lee RK, Griffith JF, Ng AW, Nung RC, Yeung DK. Wrist traction during MR arthrography improves detection of triangular fibrocartilage complex and intrinsic ligament tears and visibility of 
articular cartilage. American Journal of Roentgenology. 2016 Jan;206(1):155-61.

10. Stoller DW, editor. Magnetic resonance imaging in orthopaedics and sports medicine. Lippincott Williams \& Wilkins; 2007.

11. Hollister AM, Sanders RA, McCann S. The use of MRI in the diagnosis of an occult wrist ganglion cyst. Orthopaedic review. 1989 Nov;18(11):1210-2.

12. Vo P, Wright T, Hayden F, Dell P, Chidgey L. Evaluating dorsal wrist pain: MRI diagnosis of occult dorsal wrist ganglion. The Journal of hand surgery. $1995 \mathrm{Jul}$ 1;20(4):667-70.

13. Povlsen, Will RC Peckett B. Arthroscopic findings in patients with painful wrist ganglia. Scandinavian journal of plastic and reconstructive surgery and hand surgery. 2001 Jan 1;35(3):323-8.

14. El-Noueam KI, Schweitzer ME, Blasbalg R, Farahat AA, Culp RW, Osterman LA, Malik A. Is a Subset of Wrist Ganglia the Sequela of Internal Derangements of the Wrist Joint? MR Imaging Findings 1. Radiology. 1999 Aug;212(2):537-40.

15. Anderson SE, Steinbach LS, Stauffer E, Voegelin E. MRI for differentiating ganglion and synovitis in the chronic painful wrist. American Journal of Roentgenology. 2006 Mar;186(3):812-8. 